\title{
Micro-Opto-Mechanical Disk for Inertia Sensing
}

\author{
Ghada H. DUSHAQ*, Tadesse MULUGETA, and Mahmoud RASRAS \\ Department of Electrical Engineering and Computer Science, iMicro Center, MASDAR Institute, Building A1, PO Box \\ 54224, Masdar City, United Arab Emirates \\ *Corresponding author: Ghada H. DUSHAQ E-mail: gdushaq@masdar.ac.ae
}

\begin{abstract}
An optically enabled $z$-axis micro-disk inertia sensor is presented, which consists of a disk-shaped proof mass integrated on top of an optical waveguide. Numerical simulations show that the optical power of laser beam propagating in a narrow silicon nitride $\left(\mathrm{Si}_{3} \mathrm{~N}_{4}\right)$ waveguide located under the disk is attenuated in response to the vertical movement of the micro-disk. The high leakage power of the TM mode can effectively be used to detect a dynamic range of $1 \mathrm{~g}-10 \mathrm{~g}\left(\mathrm{~g}=9.8 \mathrm{~m} / \mathrm{s}^{2}\right)$. At lest, the waveguide is kept at a nominal gap of $1 \mu \mathrm{m}$ from the proof mass. It is adiabatically tapered to a narrow dimension of $W \times H=350 \times 220 \mathrm{~nm}^{2}$ in a region where the optical mode is intended to interact with the proof mass. Furthermore, the bottom cladding is completely etched away to suspend the waveguide and improve the optical interaction with the proof mass. The proposed optical inertia sensor has a high sensitivity of $3 \mathrm{~dB} / \mathrm{g}$ when a $50 \mu \mathrm{m}$-long waveguide is used (normalized sensitivity $0.5 \mathrm{~dB} / \mu \mathrm{m}^{2}$ ) for the vertical movement detection.
\end{abstract}

Keywords: Micro-opto-Mechanical system; photonic inertia sensor; hybrid integration

Citation: Ghada H. DUSHAQ, Tadesse MULUGETA, and Mahmoud RASRAS, "Micro-Opto-Mechanical Disk for Inertia Sensing," Photonic Sensors, 2016, 6(1): 78-84.

\section{Introduction}

Silicon micro-electro-mechanical systems (MEMS) devices are widely used for inertia and pressure sensing applications. Traditional MEMS inertia sensors employ large proof mass attached to springs which yield resonant frequency of few kilohertz $[1,2]$. A variety of transduction mechanism has been used for sensing the proof mass displacement, which includes piezoresistive [3], tunneling [4], thermal [5], capacitive [6], and optical [7]. Optically enabled micro-accelerometers can offer high resolution detection and improve sensitivity. They are also immune to electromagnetic interference and have the potential for being integrated with electronics on the same silicon platform [1]. Such a platform can provide compact device size in addition to a low fabrication cost when produced in mass. They have been used in a wide range of applications including biomedical, industrial processes such as robotics, humanactivities monitoring, and consumer electronics [8].

Typically, accelerometers quality can be specified by their sensitivity, maximum operation range, frequency response, resolution, off-axis sensitivity, and shock survival. In addition, a trade-off between the sensor's sensitivity and bandwidth should be attained. For example, low resonance frequency yields large displacements, hence resulting in a good sensor resolution but restricting its bandwidth. Capacitive accelerometer can eliminate the trade-off between the sensitivity and bandwidth by implementing a feedback circuit [9]. On the other hand, optically enabled inertia

Received: 3 November 2015 / Revised: 19 November 2015

(C) The Author(s) 2015. This article is published with open access at Springerlink.com

DOI: $10.1007 / \mathrm{s} 13320-015-0294-4$

Article type: Regular 
sensors are able to achieve sub $\mathrm{nm} / \mathrm{g}$ resolution with smaller masses [1, 2]. However, those optical detection based systems employ optical resonators or photonics crystal cavities with a narrow transmission bandwidth. Therefore, they require tunable lasers with complex control of their resonance wavelength to align with that of the optical resonator. Consequently, these systems are complex in nature. In our system, we are addressing this issue by using only passive optical components as discussed in the optical configuration section of this paper.

In this work, we propose an optically-enabled micro-disk inertia sensor. The suspended disk shape proof mass has flexibility to move in three dimensions (3-axis) with the main focus placed on the out-of-plane movement. The movement is detected by placing birefringent waveguide under the proof mass separated by an air gap. The proof mass is designed using an inertial measurement unit (IMU) platform while the waveguide is in silicon photonics ( $\mathrm{SiPh}$ ). The proposed proof mass structure is suspended using four serpentine springs. The main focus of this work is placed on the detection of the out-of-plane displacement. Therefore, the serpentine springs are designed to provide a low spring constant and optimized to allow the maximum displacement in the out-of-plane direction. This movement is detected using birefringent suspended waveguides hybrid integrated under the proof mass. They are designed using a relatively low-index- contrast silicon nitride $\left(\mathrm{Si}_{3} \mathrm{~N}_{4}\right)$ waveguides where they can carry either transverse electric (TE) or transverse magnetic (TM) optical polarizations. Furthermore, the system is based on the detection of light intensity transmission modulation in a passive waveguide, therefore, it eliminates the tedious tuning of optical resonators which significantly simplify the detection method, and in addition low cost lasers can be used. The details of sensor mechanical design are described in Section 2.1. Section 2.2 explains the optical configuration optimized for the maximum sensitivity in the out of plane movement of the inertia disk.

\section{Inertia sensor mechanical \& optical configuration}

Inertia sensors generally consist of proof mass suspended by beams which are anchored to a fixed frame and this system can be modeled by the second-order mass-damper-spring system. The out-of-plane ( $z$ ) movement is detected by two sets of nano-photonic waveguides which are placed under the proof mass. Figure 1(a) shows a schematic of the waveguide designed using a relatively low index-contrast silicon nitride $\left(\mathrm{Si}_{3} \mathrm{~N}_{4}\right)$ platform [10]. In the proposed hybrid integrated platform, the silicon on insulator (SOI) wafer can be bonded to an IMU wafer where the initial gap between the two wafers is $1 \mu \mathrm{m}$ as shown in Fig. 1(b).

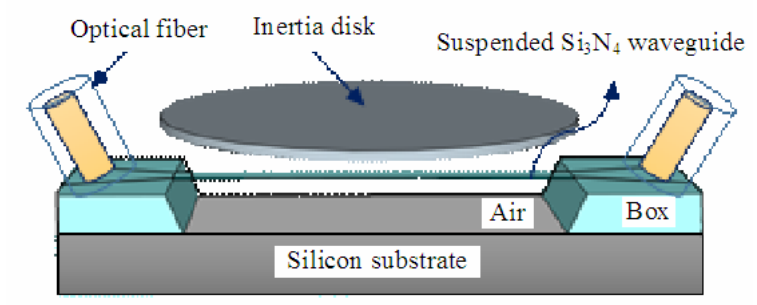

(a)

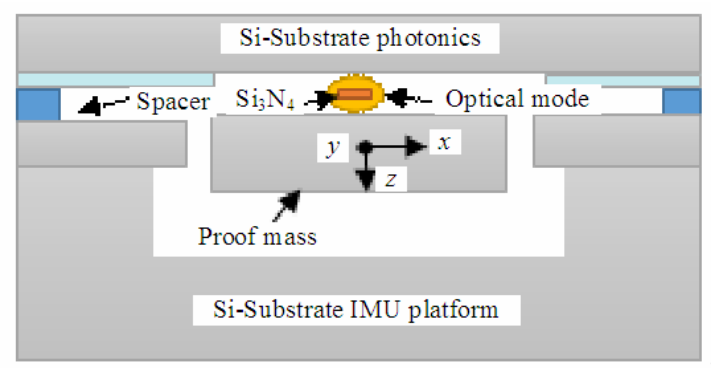

(b)

Fig. 1 Schematic representation of the proposed design of the inertia sensor: (a) a cartoon of the silicon nitrite suspended waveguide and (b) hybrid integration of SOI and IMU platform with initial gap of $1 \mu \mathrm{m}$.

\subsection{Mechanical model}

Figure 2 shows a schematic of the proposed inertia sensor. A classic serpentine springs are adopted in this design, which can offer a low spring constant and reasonable occupation area, furthermore they can be used for the in-plane as well as the out of plane displacements and they can behave like torsional spring. It is shown that the 
resonant frequency of the serpentine springs design is completely independent of residual stress value, while there is a large stress dependence for simple straight torsional rods with the same spring constant $[11,12]$. The stiffness of serpentine springs and other beams shape can be calculated based on the standard small displacement beam theory as derived in $[13,14]$.

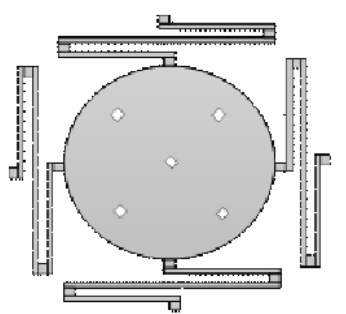

(a)

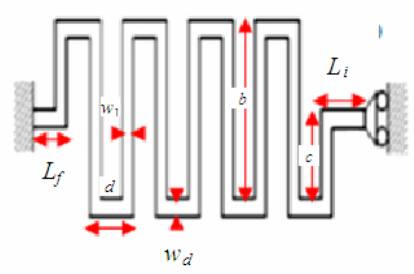

(b)
Fig. 2 Inertia sensor mechanical body: (a) schematic representation of the proposed inertia sensor and (b) serpentine spring schematic.

The static, the modal analysis, and the transient response of the inertia sensor were simulated using COMSOL Multi-Physics software. The design parameters of the sensor are summarized in Table 1. Figures 3(a), 3(b), and 3(c) illustrate the first three vibration modes of the sensor and their corresponding resonance frequency values. The three calculated fundamental vibration modes are $2.1 \mathrm{kHz}, 3.6 \mathrm{kHz}$, and $3.6 \mathrm{kHz}$. The maximum displacement of the disk in the out-of-plane (z-direction) and in-plane $(x-y \quad$ direction $)$ is calculated using a body load model ranging from $1 \mathrm{~g}$ to $10 \mathrm{~g}$. Figure 3(d) shows the maximum displacement of the sensor when a body force is acting on $z$-direction, the displacement values in the $z$-direction have the highest values which are consistent with the modal analysis results. This shows that the easiest energy of the system is in the $z$-direction, in addition it indicates a very tiny displacement in the in-plane direction under this $z$-loaded force recording $\sim 1.3 \%$ cross axis sensitivity. Since the differential gap between the disk and the waveguides is restricted to $1 \mu \mathrm{m}$, by extrapolating the gap spacing to $16 \mathrm{~g}$ in Fig. 3(d), the disk displacement is $Z=1 \mu \mathrm{m}$ which corresponds to a zero spacing between the disk and waveguide. Therefore, $16 \mathrm{~g}$ is the highest possible operational dynamic range of the sensor. From these numerical results, we choose the $1 g-10 g$ as our sensor dynamic range to operate the system safely and avoid any collapse or stiction. Figure 3(e) shows the maximum displacement of the sensor when it is loaded by an in-plane force, clearly the displacement values are very small and consistent with the modal analysis which gives the $z$-direction the maximum displacement value, hence this work focuses only on the $z$-displacement detection of an inertia sensor.
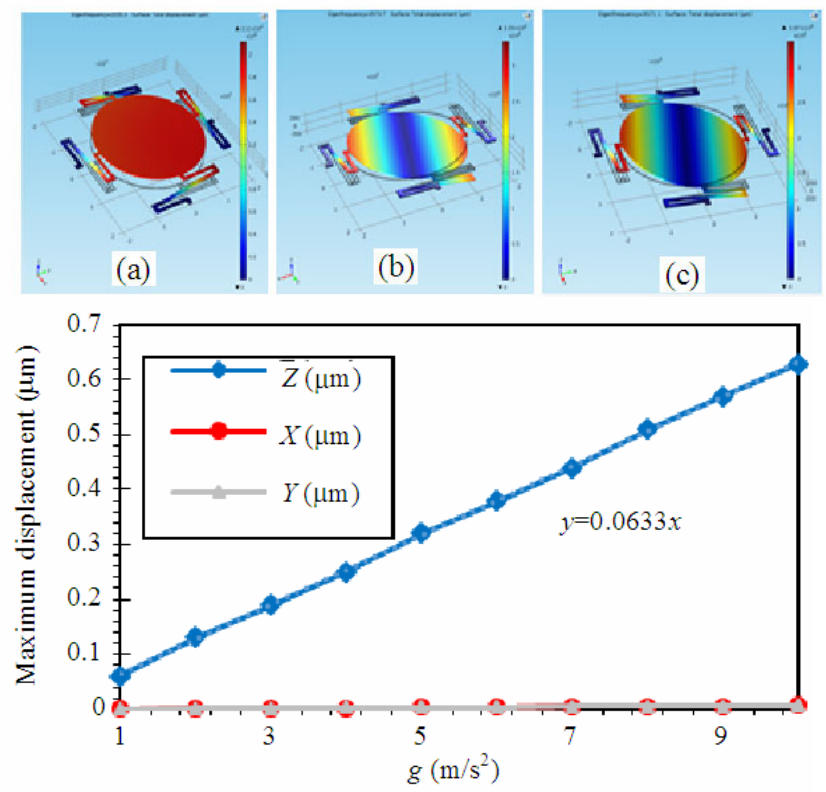

(d)

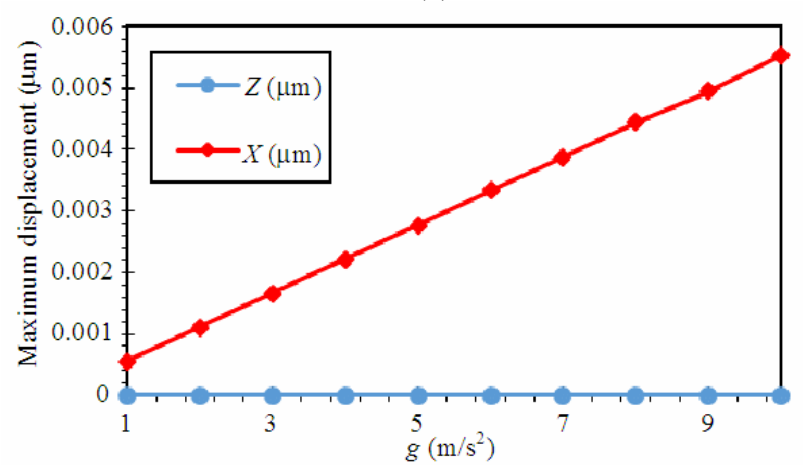

(e)

Fig. 3 Vibrational and steady state analysis of the sensor: (a) mode 1 with $2.1 \mathrm{kHz}$, (b) mode 2 with $3.6 \mathrm{kHz}$, (c) mode 3 with $3.6 \mathrm{kHz}$, (d) the maximum displacement of out-of-plane loaded inertia sensor, and (e) the maximum displacement of in-plane loaded inertia sensor. 
Table 1 Design parameters of the proposed inertia sensor.

\begin{tabular}{|c|c|c|}
\hline Design parameter & Value $(\mu \mathrm{m})$ & Expression \\
\hline$L_{i}$ & 100 & $\begin{array}{l}\text { The length of the initial part of the } \\
\text { serpentine }\end{array}$ \\
\hline$w_{i}$ & 100 & $\begin{array}{l}\text { The width of the initial part of the } \\
\text { serpentine }\end{array}$ \\
\hline$L_{f}$ & 100 & $\begin{array}{l}\text { The length of the final part of the } \\
\text { serpentine }\end{array}$ \\
\hline$w_{f}$ & 100 & $\begin{array}{l}\text { The width of the final part of the } \\
\text { serpentine }\end{array}$ \\
\hline$w_{l}$ & 80 & The width of the beam \\
\hline$d$ & 260 & The turn length \\
\hline$b$ & 2000 & The beam length \\
\hline$c$ & 1000 & $c=b / 2$ \\
\hline$n$ & 4 & Number of turns \\
\hline$t$ & 30 & $\begin{array}{l}\text { The thickness of the whole } \\
\text { structure specified by the } \\
\text { technology }\end{array}$ \\
\hline$R$ & 1500 & Proof mass radius \\
\hline
\end{tabular}

The modal analysis of the suspended $\mathrm{Si}_{3} \mathrm{~N}_{4}$ beam is also carried out and the first three vibrational modes with their corresponding resonance frequencies are shown in Fig. 4. The beam has a vibrational mode in $\mathrm{MHz}$ range which makes it very rigid to perform any displacement in the in-plane $(X-Y)$ or out of plane $(Z)$ directions. Furthermore, under an external inertia force equivalent to $10 \mathrm{~g}$, the calculated displacement in the Z-direction of the beam is $\sim 4 \mathrm{pm}$, with a tiny displacement. Therefore, the suspended waveguide is mechanically stable. Furthermore, no fluctuation due to the optical forces can affect the system performance.
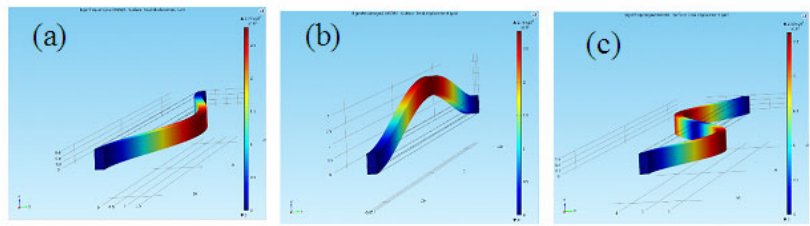

Fig. 4 The first three vibrational modes of the suspended beam $\left(\mathrm{Si}_{3} \mathrm{~N}_{4}\right.$ waveguide): (a) Mode 1 with $1 \mathrm{MHz}$, (b) Mode 2 with $2.2 \mathrm{MHz}$ and (c) Mode 3 with $2.8 \mathrm{MHz}$.

\subsection{Time response}

Logarithmic decrement approach has been used to give an approximation of the sensor damping and quality factor. The approach depends on measuring the transient response of the structure when subjected to a sudden acceleration. Figure 5 shows the $z$-displacement of the proof mass center as a function of time when a rectangle pulse of $0.1 \mathrm{~ms}$ width is applied to the sensor under atmospheric condition (an air box surrounded with the structure is designed and fluid-mechanics interaction is detected in the sensor area). By measuring the ratio of any two successive amplitudes ( $X_{1}$ and $X_{2}$ time difference) as shown in Fig. 5, the logarithmic decrement $(\delta)$ is calculated. Then, it can be shown that the damping ratio $(\zeta)$ is calculated as $[15,16]$

$$
\zeta=\frac{\delta}{\sqrt{\delta^{2}+4 \pi^{2}}} .
$$

By plotting $\lg \left(X_{j}\right)$ vs $j$ where $j=1,2, \cdots$, the slope $\delta$ is calculated as 0.4 . By substituting $\delta$ value in (1), this gives $\zeta=0.07$, therefore the system is underdamped with a quality factor $Q=\frac{1}{2 * \zeta} \sim 7$. The proof mass settles after $0.25 \mathrm{~ms}$ which gives it a possibility to be implemented in vibrating analysis devices.

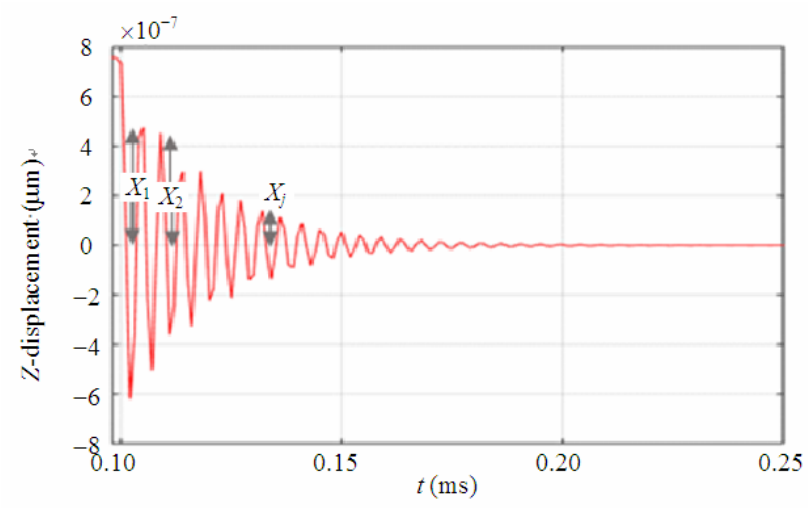

Fig. 5 Time response of inertia sensor under a sudden acceleration pulse of width $0.1 \mathrm{~ms}$.

\subsection{Optical model}

The optical waveguides are designed using relatively low-index-contrast $\mathrm{Si}_{3} \mathrm{~N}_{4}$ waveguides. This optical structure is flip-chipped on top of the IMU proof mass. In this configuration, the evanescent field of the optical waveguide interacts with the top surface of the proof mass. The larger the interaction of the optical fields with the proof mass is, the greater the scattering of the optical mode in the waveguide is, which will result in an attenuation of the optical signal.

As the mass vibrates in the out-of-plane dimensions, it will get closer or far away from the 
waveguide. This vibration can be detected as a modulation of the optical signal intensity. To maximize the interaction between the two platforms, the width of the waveguide is reduced, and the bottom $\mathrm{SiO}_{2}$ cladding is completely etched away below the waveguide leaving a suspended $\mathrm{Si}_{3} \mathrm{~N}_{4}$ with a top $\mathrm{SiO}_{2}$ cladding structure. In this layout, $\mathrm{Si}_{3} \mathrm{~N}_{4}$ waveguide has cross-section dimensions of $W \times H=350 \times 220 \mathrm{~nm}^{2}$. The oxide box thickness is $2 \mu \mathrm{m}$. Figure 6 shows the mode shapes TE and TM of waveguide with $W=0.35 \mu \mathrm{m}$.

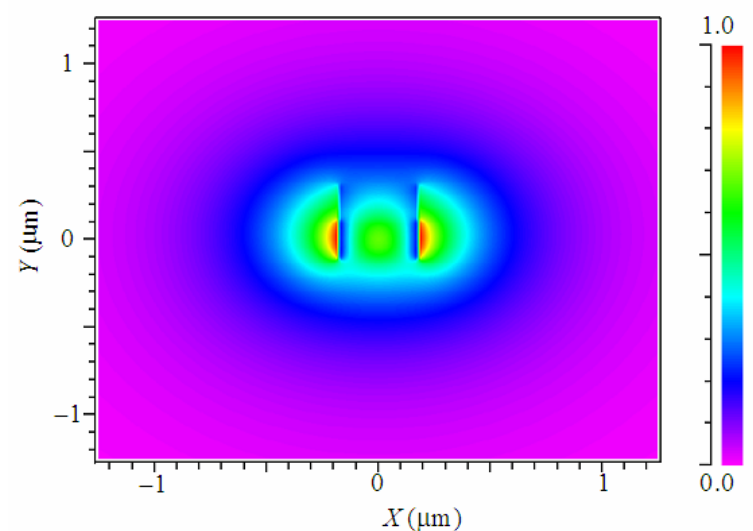

(a)

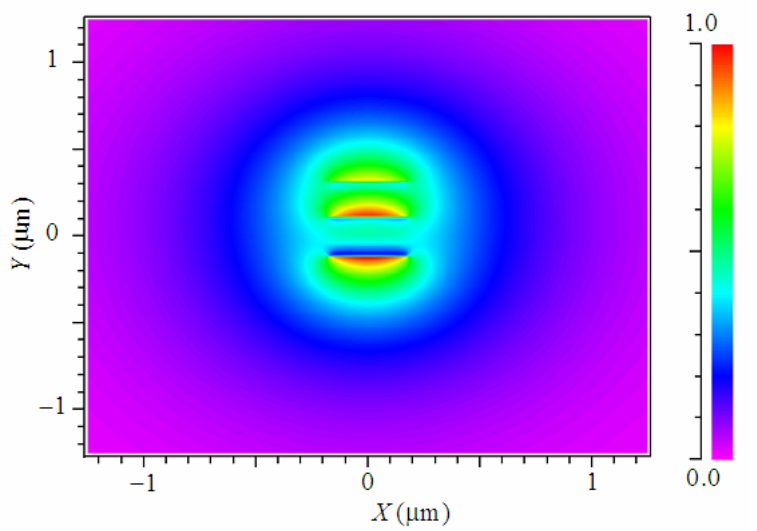

(b)

Fig. 6 Mode shapes of $0.35 \mu \mathrm{m}$ width $\mathrm{Si}_{3} \mathrm{~N}_{4} / \mathrm{SiO}_{2}$ waveguide: (a) TE mode and (b) TM mode.

\section{Results and discussion}

Numerical simulations are used to compute the leakage of the TE and TM polarizations propagating in a $50 \mu \mathrm{m}$-long waveguide as a function of gap between the two wafers and for a scan of waveguide width. Simulation results of the sensitivity of the out-of-plane disk movement are shown in Fig. 7. The sensitivity of our device is defined as the attenuation of light signal due to the mechanical movement of the disk. From Fig. 7, it can be seen that TE and TM modes have clearly distinct sensitivity behaviors, and a high detection capability up to $25 \mathrm{~dB} / \mu \mathrm{m}$ (or normalized sensitivity $0.5 \mathrm{~dB} / \mu \mathrm{m}^{2}$ ) can be achieved by using a TM mode and a narrow waveguide with a width $0.25 \mu \mathrm{m}$. In both polarizations, the sensitivity has a low value for a gap more than $1 \mu \mathrm{m}$, however as the disk becomes closer to the waveguide with gap spacing below $1 \mu \mathrm{m}$, the TM mode becomes highly sensitive, which is also possible to calculate the gap spacing by monitoring the intensity of each polarization or the ratio between them.

Note that, in practice, a tap from the light source $(\sim 6 \%)$ can be used as a monitor of the actual optical power lunched from the laser. The variation of the signal at the output of the accelerometer waveguide due to the disk displacement is then compared to this reference monitor measurement.

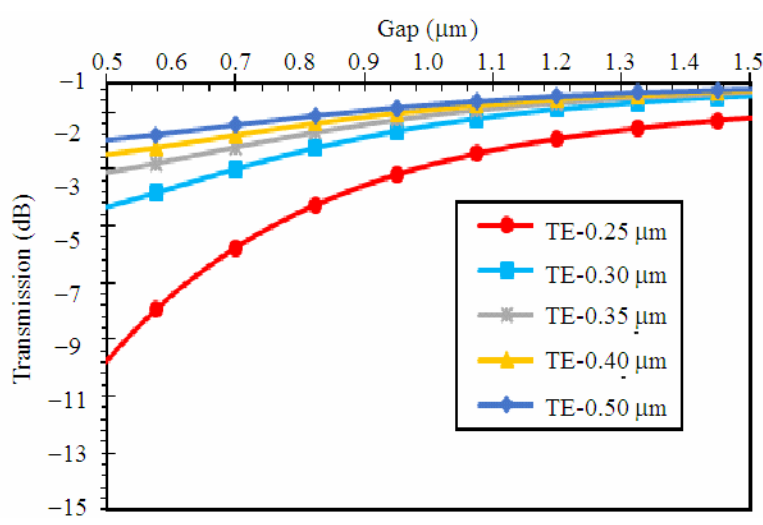

(a)

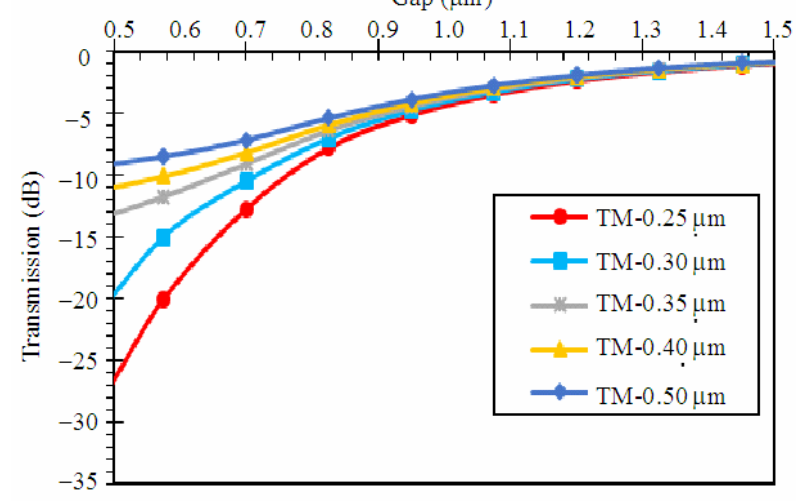

(b)

Fig. 7 Power leakage of $\mathrm{Si}_{3} \mathrm{~N}_{4}$ waveguide modes at waveguide length of $50 \mu \mathrm{m}$ : (a) TE (b) TM. 
Figure 8 shows the TE and TM modes of $0.35 \mu \mathrm{m}$ waveguide width, the gap scan is reduced to $1.1 \mu \mathrm{m}$ to define the operational regime of the device where the sensitivity detection is the maximum. In addition, it can be seen that the range is approximately linear which makes it an optimum choice for the readout circuit in an experimental setup. From fabrication, perspective wider waveguide with $0.35 \mu \mathrm{m}$ width has more mechanical stability and offers high sensitivity values.

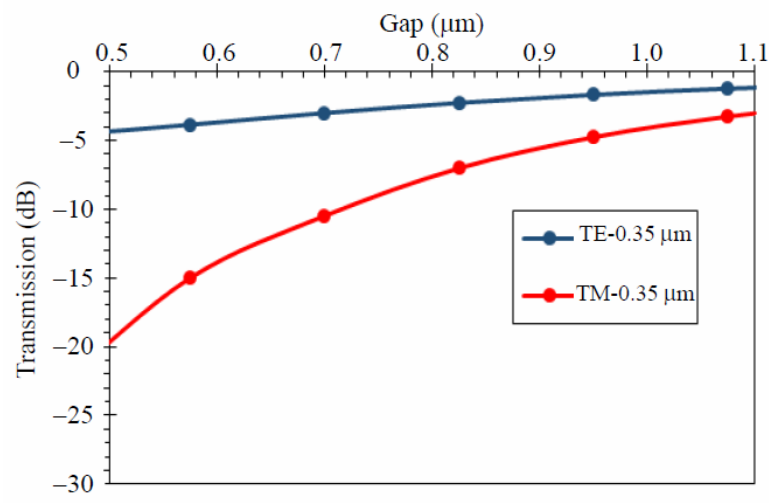

Fig. 8 Power leakage of TE and TM modes of $0.35 \mu \mathrm{m}$ width $\mathrm{Si}_{3} \mathrm{~N}_{4}$ waveguide.

TE mode can also be used for narrow gap detection where the waveguide is designed to be longer than $50 \mu \mathrm{m}$. The power leakage as a function of waveguide length for both $0.5 \mu \mathrm{m}$ and $1 \mu \mathrm{m}$ gaps of $0.35 \mu \mathrm{m}$ waveguide width is shown in Fig. 9 .

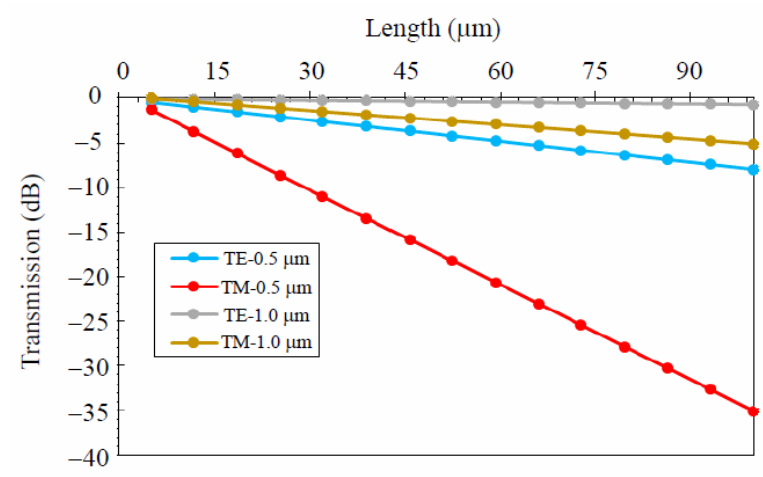

Fig. 9 Power leakage of $0.35 \mu \mathrm{m}$ width $\mathrm{Si}_{3} \mathrm{~N}_{4}$ waveguide as a function of length.

Based on the above numerical results, an optical integrated waveguide scheme for an accurate and yet a large dynamic range detection of the out-of-plane displacement is shown in Fig. 9. In this scheme, two sets of straight waveguides have been used, one of them with a TM component $\left(L \times W=50 \times 0.35 \mu \mathrm{m}^{2}\right)$ is used to detect the course movement. On the other hand, the other straight waveguide structure $(L \times W=$ $100 \times 0.35 \mu \mathrm{m}^{2}$ ) is dedicated to sense the fine movement of the disk. With this configuration, we can successfully measure a tiny displacement of $<0.05 \mu \mathrm{m}$ that corresponds to sub- $g$ resolution over $10 \mathrm{~g}$ range.

\section{Conclusions}

Optically enabled inertia sensor with a dynamic range up to $10 \mathrm{~g}$ of operation has been presented. The TE and TM light modes in relatively low-indexcontrast $\mathrm{Si}_{3} \mathrm{~N}_{4}$ suspended waveguide has been studied. The two light modes show different behaviors in the light intensity modulation, hence with this completely etched bottom cladding waveguide, TM mode is highly sensitive to any out of plane movement recording $\sim 25 \mathrm{~dB} / \mu \mathrm{m}$ change in light intensity for $0.25 \mu \mathrm{m} \mathrm{Si}{ }_{3} \mathrm{~N}_{4}$ width. The out of plane optical displacement detection and the time response behaviors of the proposed inertia sensor give the possibility to use it in the motion detection and smart user interface. In addition, low cost and high detection capability are offered in this design structure without the need to employ tunable optical resonators or photonics cavities.

\section{Acknowledgment}

This work is funded by Mubadala Development Company-Abu Dhabi, Economic Development Board-Singapore, and GLOBALFOUNDRIESSingapore under the framework of 'Twinlab' project with participation of $A * S T A R$ Institute of Microelectronics-Singapore, Masdar Institute of Science and Technology-Abu Dhabi and GLOBALFOUNDRIES-Singapore.

Open Access This article is distributed under the terms of the Creative Commons Attribution 4.0 International License (http:// creativecommons.org/ licenses/by/4.0/), which permits unrestricted use, distribution, and reproduction in any medium, provided you give 
appropriate credit to the original author(s) and the source, provide a link to the Creative Commons license, and indicate if changes were made.

\section{References}

[1] B. Dong, H. Cai, J. M. Tsai, D. L. Kwong, and A. Q. Liu, "An on-chip opto-mechanical accelerometer," in Proceedings of IEEE Conference on Micro Electro Mechanical Systems (MEMS), Taipei, pp. 641-644, 2013.

[2] K. Zandi, J. A. Belanger, and Y. A. Peter, "Design and demonstration of an in-plane silicon-on-insulator optical MEMS Fabry-perot-based accelerometer integrated with channel waveguides," Journal of Microelectromechanical Systems, 2012, 21(6): 1464-1470.

[3] H. Seidel, U. Fritsch, R. Gottinger, J. Schalk, J. Walter, and K. Ambaum, "A peiezoresistive silicon accelerometer with monolithically integrated CMOS-circuitry," in the 8th International Conference on Solid-State Sensors and Actuators, 1995 and Eurosensors IX.. Transducers '95, Stockholm, Sweden, pp. 597-600, 1995.

[4] C. Yeh and K. Najafi, "A low-voltage bulk-silicon tunneling-based microaccelerometer," in International Electron Devices Meeting, 1995, Washington, DC, pp. 593-596, 1995.

[5] A .M. Leung, J. Jones, E. Czyzewska, J. Chen, and B. Woods, "Micromachened accelerometer based on convection heat transfer," in Proceeding of IEEE Micro Electro Mechanical Systems Workshop (MEMS'98), Heidelberg, Germany, pp. 627-630, 1998 .

[6] C. Sun, C. Wang, and W. Fang, "On the sensitivity improvement of CMOS capacitive accelerometer," Sensors \& Actuators A Physical, 2008, 14(12):
347-352.

[7] D. N. Hutchison and S. A. Bhave, "Z-axis optomechanical accelerometer," in Proceeding of IEEE Conference on Micro Electro Mechanical Systems (MEMS), Paris, France, pp. 615-619, 2012.

[8] N. Yazdi, F. Ayazi, and K. Najafi, "Micromachined inertia sensors," Proceeding of the IEEE, 1998, 86(8): 1640-1659.

[9] B. E. Boser and R. T. Howe, "Surface micromachined accelerometer," IEEE Journal of Solid-State Circuits, 1996, 31(3): 366-375.

[10] J. F. Bauters, M. J. R. Heck, D. John, D. Dai, M. C. Tien, J. S. Barton, et al., "Ultra-low-loss high-aspect-ratio $\mathrm{Si}_{3} \mathrm{~N}_{4}$ waveguides," Optics Express, 2011, 19(4): 3163-3167.

[11] V. A. Aksyuk, M. E. Simon, F. Pardo, S. Arney, D. Lopez and A.Villanueva, A 2002 Optical MEMS design for telecommunications applications Solid-State Sensor, Actuator and Microsystem Workshop (Hilton Head Island, SC, 2002).

[12] G. Barillaro, A. Molfese, A. Nannini, and F. Pieri, "Analysis, simulation and relative performances of two kinds of serpentine spring," Journal of Micromechanics \& Microengineering, 2005, 15(4): 736-746.

[13] L. D. Landau, L. P. Pitaevskii, A. M. Kosevich, and E. M. Lifshitz, Theory of elasticity. Massachusetts: Addison-Wesley, Inc. Reading, 1959.

[14] G. K. Fedder, "Simulation of microelectromechanical systems," Ph.D. dissertation, Dept. University of California, Berkeley, 1994.

[15] M. I. Younis, MEMS linear and nonlinear statics and dynamics. Berlin: Springer, 2011.

[16] W. T. Thomoson and M. D Dahleh, Theory of vibration with applications. New Jersey: Prentice Hall, 1998. 